\title{
Improved WKB analysis of cosmological perturbations
}

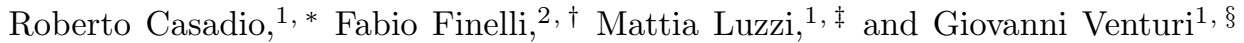 \\ ${ }^{1}$ Dipartimento di Fisica, Università di Bologna and I.N.F.N., \\ Sezione di Bologna, via Irnerio 46, 40126 Bologna, Italy \\ ${ }^{2}$ IASF/INAF, Istituto di Astrofisica Spaziale e Fisica Cosmica, \\ Istituto Nazionale di Astrofisica, Sezione di Bologna, via Gobetti 101, 40129 Bologna, Italy.
}

\begin{abstract}
Improved Wentzel-Kramers-Brillouin (WKB)-type approximations are presented in order to study cosmological perturbations beyond the lowest order. Our methods are based on functions which approximate the true perturbation modes over the complete range of the independent (Langer) variable, from sub-horizon to super-horizon scales, and include the region near the turning point. We employ both a perturbative Green's function technique and an adiabatic (or "semiclassical") expansion (for a linear turning point) in order to compute higher order corrections. Improved general expressions for the WKB scalar and tensor power spectra are derived for both techniques. We test our methods on the benchmark of power-law inflation, which allows comparison with exact expressions for the perturbations, and find that the next-to-leading order adiabatic expansion yields the amplitude of the power spectra with excellent accuracy, whereas the next-to-leading order with the perturbative Green's function method does not improve the leading order result significantly. However, in more general cases, either or both methods may be useful.
\end{abstract}

PACS numbers: 98.80.Cq, 98.80.-k

\section{INTRODUCTION}

Anisotropies in the cosmic microwave background $(\mathrm{CMB})$ radiation and inhomogeneities in the large scale structures of the Universe have nowadays become a fundamental tool to study the early universe. This trend will persist in the next years due to the data releases of Wilkinson Microwave Anisotropy Probe 1], Sloan Digital Sky Survey [2] and the launch of the Planck mission [3].

In a primordial inflationary phase, matter and spacetime fluctuations are generated which inherit characteristics from the particular inflationary model studied (for an introduction and review see, e.g. Ref. 4] ). It is therefore very important to be able to determine the power spectra of cosmological perturbations for a large variety of different inflationary models, so that comparison with available and future data will tell us which models satisfactorily represent the time evolution of the Universe. Since exact solutions are not available for cosmological perturbations in general inflationary models (except for the case of an exponential potential [5]) approximation methods are very welcome. The method of approximation most used is the slow-rollover approximation, introduced by Stewart and Lyth [6] (see Ref. 7] for a review), which was inspired by power-law inflation.

It is also worthwhile testing different approximation schemes. In this paper we present Wentzel-KramersBrillouin (WKB)-type approaches which improve the

\footnotetext{
*Electronic address: Roberto.Casadio@bo.infn.it

${ }^{\dagger}$ Electronic address: finelli@bo.iasf.cnr.it

${ }^{\ddagger}$ Electronic address: Mattia.Luzzi@bo.infn.it

$\S$ Electronic address: armitage@bo.infn.it
}

proposals of Refs. 8, 9] (see also Refs. 10, 11, 12, 13, 14, 15] for other approximations). For power-law inflation, the usual WKB method to lowest order reproduces exactly the spectral indices of perturbations, but the amplitudes are still rendered poorly. Given the importance of the consistency equation between the ratio of tensor to scalar amplitudes and the spectral index of tensor modes for single Klein-Gordon scalar field inflation [4], we believe that the prediction on the amplitudes should also be improved, as is done in Ref. 16] by means of the socalled "uniform approximation" to next-to-leading order (for the details on the formalism, see Ref. 17]).

Let us begin by recalling the Robertson-Walker metric in conformal time $\eta$,

$$
\mathrm{d} s^{2}=a^{2}(\eta)\left[-\mathrm{d} \eta^{2}+\mathrm{d} \vec{x} \cdot \mathrm{d} \vec{x}\right],
$$

where $a$ is the scale factor of the Universe. Scalar (density) and tensor (gravitational wave) fluctuations are determined on this background by two functions, respectively denoted as $\mu=\mu_{\mathrm{S}}:=a Q(Q$ is the Mukhanov variable [10]) and $\mu=\mu_{\mathrm{T}}:=a h$ ( $h$ is the amplitude of the two polarizations of gravitational waves) [18, 19], which satisfy a one-dimensional Schrödinger-like equation

$$
\left[\frac{\mathrm{d}^{2}}{\mathrm{~d} \eta^{2}}+k^{2}-U(\eta)\right] \mu=\left[\frac{\mathrm{d}^{2}}{\mathrm{~d} \eta^{2}}+\Omega^{2}(k, \eta)\right] \mu=0,
$$

where $k$ is the wave-number. The time-dependent frequency is given by

$$
\Omega^{2}(k, \eta)=k^{2}-\frac{z^{\prime \prime}}{z},
$$

where $z=z_{\mathrm{S}}:=a \phi^{\prime} / \mathcal{H}$ for scalar ( $\phi$ is the homogenous inflaton) and $z=z_{\mathrm{T}}:=a$ for tensor perturbations, primes denote derivatives with respect to $\eta$, and 
$\mathcal{H}\left(:=a^{\prime} / a\right)$ is the conformal Hubble parameter. Eq. (2) must be solved together with the condition that the modes are initially plane waves for wavelengths much shorter than the Hubble radius,

$$
\lim _{\frac{k}{a H} \rightarrow+\infty} \mu(k, \eta)=d \frac{\mathrm{e}^{-i k \eta}}{\sqrt{2 k}},
$$

where $d=d_{\mathrm{S}}:=1$ and $d=d_{\mathrm{T}}:=\sqrt{8 \pi} / m_{\mathrm{Pl}}$ for scalar and tensor perturbations ( $m_{\mathrm{Pl}}$ is the Planck mass). The dimensionless power spectra of scalar and tensor fluctuations are then given by

$$
\mathcal{P}_{\zeta}=\frac{k^{3}}{2 \pi^{2}}\left|\frac{\mu_{\mathrm{S}}}{z_{\mathrm{S}}}\right|^{2}, \quad \mathcal{P}_{h}=\frac{4 k^{3}}{\pi^{2}}\left|\frac{\mu_{\mathrm{T}}}{z_{\mathrm{T}}}\right|^{2} .
$$

The spectral indices and their runnings are defined at an arbitrary pivot scale $k_{*}$ as

$$
n_{\mathrm{S}}-1:=\left.\frac{\mathrm{d} \ln \mathcal{P}_{\zeta}}{\mathrm{d} \ln k}\right|_{k=k_{*}} \quad, \quad n_{\mathrm{T}}:=\left.\frac{\mathrm{d} \ln \mathcal{P}_{h}}{\mathrm{~d} \ln k}\right|_{k=k_{*}},
$$

and

$$
\alpha_{\mathrm{S}}:=\left.\frac{\mathrm{d}^{2} \ln \mathcal{P}_{\zeta}}{(\mathrm{d} \ln k)^{2}}\right|_{k=k_{*}}, \quad \alpha_{\mathrm{T}}:=\left.\frac{\mathrm{d}^{2} \ln \mathcal{P}_{h}}{(\mathrm{~d} \ln k)^{2}}\right|_{k=k_{*}} .
$$

The evolution of the Universe is usually described by means of a set of flow equations [8, 20, 21]. The zero horizon flow function is defined by

$$
\epsilon_{0}:=\frac{H\left(N_{i}\right)}{H(N)}
$$

where $H:=\dot{a} / a$ is the Hubble rate and dots denote derivatives with respect to cosmic time $\mathrm{d} t=a(\eta) \mathrm{d} \eta$. In this expression, $N$ is the number of e-folds, $N:=\ln \left(a / a_{i}\right)$ [where $a_{i}=a\left(\eta_{i}\right)$ ] after the arbitrary initial time $t_{i}=$ $t_{i}\left(\eta_{i}\right)$. The hierarchy of horizon flow functions is then defined according to

$$
\epsilon_{n+1}:=\frac{\mathrm{d} \ln \left|\epsilon_{n}\right|}{\mathrm{d} N}, \quad n \geq 0
$$

and we recall that inflation takes place for $\epsilon_{1}<1$.

The effective potentials $U=U_{\mathrm{S}}$ and $U=U_{\mathrm{T}}$ can now be expressed in terms of $\epsilon_{1}, \epsilon_{2}$, and $\epsilon_{3}$ only as

$$
\begin{aligned}
& \frac{U_{\mathrm{T}}(\eta)}{a^{2} H^{2}}=2-\epsilon_{1} \\
& \frac{U_{\mathrm{S}}(\eta)}{a^{2} H^{2}}=2-\epsilon_{1}+\frac{3}{2} \epsilon_{2}-\frac{1}{2} \epsilon_{1} \epsilon_{2}+\frac{1}{4} \epsilon_{2}^{2}+\frac{1}{2} \epsilon_{2} \epsilon_{3},
\end{aligned}
$$

and Eq. (2) can be solved exactly for $\epsilon_{1}$ constant in time, which is the case of an exponential potential for the inflaton. When the $\epsilon_{n}$ have an arbitrary time dependence, approximate methods are necessary.

A WKB analysis of this problem has been recently presented in Ref. [8] to first order in the adiabatic (or "semiclassical") expansion (see next Section and Ref. [22] for precise definitions), in which the Langer transformation [23]

$$
x:=\ln \left(\frac{k}{H a}\right), \quad \chi:=\left(1-\epsilon_{1}\right)^{1 / 2} \mathrm{e}^{-x / 2} \mu,
$$

introduced in Ref. [12] is used [34] in order to improve the accuracy. Such a transformation brings Eq. (2) into the form

$$
\left[\frac{\mathrm{d}^{2}}{\mathrm{~d} x^{2}}+\omega^{2}(x)\right] \chi=0
$$

where the (new) frequency $\omega(x)$ in general vanishes at the classical "turning point" $x=x_{*}$. On sub-horizon scales $\left(x \gg x_{*}\right)$, the mode $\chi$ oscillates $(\omega \simeq k)$ and the standard WKB approximation can be applied without difficulty. On super-horizon scales $\left(x \ll x_{*}\right)$, the perturbations exponentially decay or grow and the standard WKB approximation can again be applied. One then matches the two approximate solutions at $x=x_{*}$ to obtain the super-horizon amplitudes at $x_{f} \ll x_{*}$ which determine the CMB spectra. This procedure opens up the possibility of further improving the knowledge of the spectra by including subsequent adiabatic orders.

However, a straightforward generalization to higher adiabatic orders is seriously hindered by the lack of a general prescription for matching the two WKB branches with sufficient accuracy. This problem was thoroughly discussed in Refs. 23, 24], where it was also suggested to replace the standard (plane wave-like) WKB functions with Bessel's functions. The latter indeed remain good approximations at the turning point, whereas the former require the matching with yet another particular solution at some (unspecified) points both to the left and right of $x=x_{*}$. We shall follow the proposal of Ref. [23] for a linear turning point and show that the inclusion of higher adiabatic orders indeed improve the results, contrarily to what was found in Ref. 25]. We shall also introduce a new (perturbative) expansion for general turning point, which makes use of the Green's function technique, and compare the corresponding corrections for the case of power law inflation.

The article is organized as follows: In Section [1] we shall review the application to inflationary cosmological perturbations of the standard WKB approximation to leading adiabatic order [8] and its shortcomings. In Section III we shall describe in full generality a method of improving the results to higher orders, both by the perturbative expansion discussed in detail in Section IV and the adiabatic expansion reviewed in Section $\nabla$ In Section VI we shall apply the improved WKB approximation to power-law inflation which, being exactly solvable, will allow us to test the improved WKB predictions against exact solutions. Finally, we shall give our conclusions and observations in Section VII 


\section{STANDARD WKB APPROXIMATION}

In this Section we begin by recalling that the straightforward application of the standard WKB method leads to a poor approximation and that the results can be improved to first adiabatic order by means of the transformation (9), as was shown in Ref. 8]. However, the extension to higher orders is more subtle, as we shall discuss in some detail.

The WKB approximation is defined by first introducing a "small" parameter $\delta>0$ in order to perform the formal adiabatic (or semiclassical) expansion of the mode functions 22] 35]. Consequently, Eq. (2) is formally replaced by

$$
\left[\delta \frac{\mathrm{d}^{2}}{\mathrm{~d} \eta^{2}}+\Omega^{2}(k, \eta)\right] \mu=0
$$

and, of course, the limit $\delta \rightarrow 1$ must be taken at the end of the computation. We then denote the leading order term in such an expansion of $\mu$ by

$$
\mu_{\mathrm{WKB}}(k, \eta)=\frac{\mathrm{e}^{ \pm \frac{i}{\sqrt{\delta}} \int \eta} \Omega(k, \tau) \mathrm{d} \tau}{\sqrt{2 \Omega(k, \eta)}}
$$

which satisfies the following differential equation

$$
\left[\delta \frac{\mathrm{d}^{2}}{\mathrm{~d} \eta^{2}}+\Omega^{2}(k, \eta)-\delta Q_{\Omega}(k, \eta)\right] \mu_{\mathrm{WKB}}=0,
$$

where

$$
Q_{\Omega}(k, \eta):=\frac{3}{4} \frac{\left(\Omega^{\prime}\right)^{2}}{\Omega^{2}}-\frac{\Omega^{\prime \prime}}{2 \Omega}
$$

We now observe that $\mu_{\mathrm{WKB}}$ is the exact solution of Eq. (11) in the "adiabatic limit" $\delta \rightarrow 0$, and is expected to be a good approximation to the exact $\mu$ (in the limit $\delta \rightarrow 1)$ if

$$
\Delta:=\left|\frac{Q_{\Omega}}{\Omega^{2}}\right| \ll 1
$$

On using the potentials in Eqs. (8) in terms of $a$ and $H$, one finds

$$
\Delta_{\mathrm{S}, \mathrm{T}}= \begin{cases}\left(\frac{a H}{k}\right)^{4} \mathcal{O}\left(\epsilon_{n}\right) \sim 0 & \text { sub-horizon scales } \\ \frac{1}{8}+\mathcal{O}\left(\epsilon_{n}\right) & \text { super-horizon scales. }\end{cases}
$$

The result for super-horizon scales suggests that a change of variable and function are necessary in order to obtain better accuracy.

In fact, on employing the transformation (9), one finds (for $\delta=1$ ) the new equations of motion (10), which explicitly read

$$
\begin{aligned}
& \frac{\mathrm{d}^{2} \chi_{\mathrm{S}}(x)}{\mathrm{d} x^{2}}+\left[\frac{\mathrm{e}^{2 x}}{\left(1-\epsilon_{1}\right)^{2}}-\frac{1}{4}\left(\frac{3-\epsilon_{1}}{1-\epsilon_{1}}\right)^{2}-\frac{\left(3-2 \epsilon_{1}\right) \epsilon_{2}}{2\left(1-\epsilon_{1}\right)^{2}}-\frac{\left(1-2 \epsilon_{1}\right) \epsilon_{2} \epsilon_{3}}{2\left(1-\epsilon_{1}\right)^{3}}-\frac{\left(1-4 \epsilon_{1}\right) \epsilon_{2}^{2}}{4\left(1-\epsilon_{1}\right)^{4}}\right] \chi_{\mathrm{S}}(x)=0 \\
& \frac{\mathrm{d}^{2} \chi_{\mathrm{T}}(x)}{\mathrm{d} x^{2}}+\left[\frac{\mathrm{e}^{2 x}}{\left(1-\epsilon_{1}\right)^{2}}-\frac{1}{4}\left(\frac{3-\epsilon_{1}}{1-\epsilon_{1}}\right)^{2}+\frac{\epsilon_{1} \epsilon_{2}}{2\left(1-\epsilon_{1}\right)^{2}}+\frac{\epsilon_{1} \epsilon_{2} \epsilon_{3}}{2\left(1-\epsilon_{1}\right)^{3}}+\frac{\left(2+\epsilon_{1}\right) \epsilon_{1} \epsilon_{2}^{2}}{4\left(1-\epsilon_{1}\right)^{4}}\right] \chi_{\mathrm{T}}(x)=0
\end{aligned}
$$

From $\omega_{\mathrm{S}}^{2}(x)$ and $\omega_{\mathrm{T}}^{2}(x)$, respectively given by the expressions in the square brackets of Eqs. (17a) and (17b), we now obtain

$$
\begin{cases}\Delta_{\mathrm{S}, \mathrm{T}}=e^{-2 x} \mathcal{O}\left(\epsilon_{n}\right) \sim 0 & \text { sub-horizon scales } \\ \Delta_{\mathrm{S}}=\frac{4}{27}\left(\epsilon_{1} \epsilon_{2}^{2}+\epsilon_{1} \epsilon_{2} \epsilon_{3}+\frac{\epsilon_{2} \epsilon_{3}^{2}}{2}+\frac{\epsilon_{2} \epsilon_{3} \epsilon_{4}}{2}\right)+\mathcal{O}\left(\epsilon_{n}^{4}\right) & \text { super-horizon scales } \\ \Delta_{\mathrm{T}}=\frac{4}{27}\left(\epsilon_{1} \epsilon_{2}^{2}+\epsilon_{1} \epsilon_{2} \epsilon_{3}\right)+\mathcal{O}\left(\epsilon_{n}^{4}\right) & \text { super-horizon scales }\end{cases}
$$

and it is thus obvious that $\Delta$ becomes very small for any inflationary potential satisfying the slow-roll conditions [12] in both limits of interest (but, remarkably, not at the turning point). As a consequence, the WKB approximation is now also valid on super-horizon scales.

One might naively expect that the transformation (9) will improve the WKB approach to all orders (in the formal expansion in $\delta$ ), thus yielding increasingly better results. However, since $Q$ diverges at the turning point $x=x_{*}$, whether the function $\mu_{\mathrm{WKB}}$ can still be regarded as a solution to Eq. (11) for $x \sim x_{*}$ in the adiabatic limit $\delta \rightarrow 0$ becomes a subtle issue. It is precisely for 
this reason that one usually considers particular solutions around the turning point (for example, combinations of Airy functions for a linear turning point) which must then be matched with the asymptotic form (12) on both sides of the turning point. The problem with this standard procedure is that there is no general rule to determine the actual matching points (which cannot be $x_{*}$ where $Q$ diverges) in such a way that the overall error be small. As a consequence, the standard adiabatic expansion for $\delta \ll 1$ may not lead to a reliable approximation around the turning point, which would then result in large errors to higher orders and a questionable further extension of all the expressions to the interesting case $\delta=1$.

It was in fact shown in Ref. 25 that a simple extension to higher orders of the above procedure [26] actually seems to reduce the accuracy with respect to the leading order results of Ref. 8]. We shall see in the next Section that one can overcome this problem by introducing mode functions which remain good approximations also around the turning point.

\section{IMPROVED WKB APPROXIMATION}

We shall now describe a method to improve the WKB approximation to all orders by following the scheme outlined in Ref. [23] (see also Ref. [27]). The basic idea is to use approximate expressions which are valid for all values of the coordinate $x$ and then expand around them. Two different such expansions we shall consider, namely one based on the Green's function technique and the other on the usual adiabatic expansion [23].

Let us first illustrate the main idea of Ref. 23] for the particular case $\omega^{2}=C\left(x-x_{*}\right)^{n}$, with $C$ a positive costant. All the solutions to Eq. (10) for $x>x_{*}$ can be written as linear combinations of the two functions

$$
u_{ \pm}(x)=\sqrt{\frac{\xi(x)}{\omega(x)}} J_{ \pm m}[\xi(x)]
$$

where

$$
\xi(x):=\int_{x_{*}}^{x} \omega(y) \mathrm{d} y, \quad m=\frac{1}{n+2},
$$

and $J_{\nu}$ are Bessel functions 28]. Moreover, for a general frequency $\omega$, the expressions in Eq. (19) satisfy

$$
\left[\frac{\mathrm{d}^{2}}{\mathrm{~d} x^{2}}+\omega^{2}(x)-\sigma(x)\right] u_{ \pm}=0
$$

where the quantity (primes from here on will denote derivatives with respect to the argument of the given function)

$$
\begin{aligned}
\sigma(x) & =\frac{3}{4} \frac{\left(\omega^{\prime}\right)^{2}}{\omega^{2}}-\frac{\omega^{\prime \prime}}{2 \omega}+\left(m^{2}-\frac{1}{4}\right) \frac{\omega^{2}}{\xi^{2}} \\
& =Q_{\omega}+\left(m^{2}-\frac{1}{4}\right) \frac{\omega^{2}}{\xi^{2}}
\end{aligned}
$$

contains the term $Q$ defined in Eq. (14), whose divergent behavior at the turning point $x=x_{*}$ we identified as the possible cause of failure of the standard WKB approach. For a general (finite) frequency, which can be expanded in powers of $x-x_{*}$ [36],

$$
\omega^{2}(x)=C\left(x-x_{*}\right)^{n}\left[1+\sum_{q \geq 1} c_{q}\left(x-x_{*}\right)^{q}\right]
$$

one finds that the extra term in Eq. (22) precisely "removes" the divergence in $Q$ at the turning point. In fact, the residue

$$
\lim _{x \rightarrow x_{*}} \sigma(x)=\frac{3(n+5) c_{1}^{2}}{2(n+4)(n+6)}-\frac{3 c_{2}}{n+6},
$$

is finite [23, 27]. The finiteness of $\sigma$ at the turning point is crucial in order to extend the WKB method to higher orders (this point was missed in Ref. 25]). It is also easy to show that, for the cases of interest, $\left|\sigma / \omega^{2}\right| \simeq \Delta$ as given in Eq. (18) for $x \rightarrow \pm \infty$, so that the new approximate solutions remain very accurate for large $|x|$. For slowly varying frequencies, with $|\sigma(x)|$ small everywhere, the functions (19) are thus expected to be good approximations to the solutions of Eq. (10) for the whole range of $x$, including the turning point.

We can now introduce both the adiabatic expansion of the previous Section and a new formal expansion of the mode functions by replacing Eq. (10) by

$$
\left[\delta \frac{\mathrm{d}^{2}}{\mathrm{~d} x^{2}}+\omega^{2}(x)-\delta \sigma(x)\right] \chi=-\delta \varepsilon \sigma(x) \chi,
$$

where $\delta$ and $\varepsilon$ are "small" positive parameters. We shall refer to expressions proportional to $\delta^{n}$ as the $n$-th adiabatic order and to those proportional to $\varepsilon^{n}$ as the $n$-th perturbative order.

It is convenient to consider the solutions to Eq. (25) to the left and right of the turning point $x_{*}$ separately. We shall call:

region I) where $\omega^{2}>0$ (on the right of $x_{*}$ ), with

$$
\omega_{\mathrm{I}}(x):=\sqrt{\omega^{2}(x)}, \quad \xi_{\mathrm{I}}(x):=\int_{x_{*}}^{x} \omega_{\mathrm{I}}(y) \mathrm{d} y,
$$

and the solutions to the corresponding homogeneous equation (21) are given in Eq. (19);

region II) where $\omega^{2}<0$ (on the left of $x_{*}$ ), with

$$
\omega_{\mathrm{II}}(x):=\sqrt{-\omega^{2}(x)}, \quad \xi_{\mathrm{II}}(x):=\int_{x}^{x_{*}} \omega_{\mathrm{II}}(y) \mathrm{d} y,
$$

and the solutions to the corresponding homogeneous equation (21) are obtained from those in Eq. (19) by replacing the $J_{\nu}$ with the $I_{\nu}$ [28]. 
Corresponding expressions are obtained for all relevant quantities, for example $\sigma_{\mathrm{I}}$ and $\sigma_{\mathrm{II}}$, and we shall omit the indices I and II whenever it is not ambiguous.

Although it is possible to expand in both parameters, so as to obtain terms of order $\delta^{p} \varepsilon^{q}$ (with $p$ and $q$ positive integers), in the following we shall just consider each expansion separately, that is we shall set $\delta=1$ in the perturbative expansion (see Section IV) and $\varepsilon=1$ in the adiabatic expansion (see Section $\nabla$ ).

\section{PERTURBATIVE EXPANSION}

For $\delta=1$, the limit $\varepsilon \rightarrow 0$ is exactly solvable and yields the solutions (19), whereas the case of interest (10) is recovered in the limit $\varepsilon \rightarrow 1$, which must always be taken at the end of the computation. The solutions to Eq. (25) can be further cast in integral form as

$$
\chi(x)=u(x)-\varepsilon \int G(x, y) \sigma(y) \chi(y) \mathrm{d} y,
$$

where $u(x)=A_{+} u_{+}(x)+A_{-} u_{-}(x)$ is a linear combination of solutions (19) to the corresponding homogeneous equation (21), and $G(x, y)$ is the Green's function determined by

$$
\left[\frac{\mathrm{d}^{2}}{\mathrm{~d} x^{2}}+\omega^{2}(x)-\sigma(x)\right] G(x, y)=\varepsilon \delta(x-y) .
$$

The solutions of the homogeneous equation (21) in the two regions are then given by

$$
\begin{aligned}
u_{\mathrm{I}}(x) & =\sqrt{\frac{\xi_{\mathrm{I}}(x)}{\omega_{\mathrm{I}}(x)}}\left\{A_{+} J_{+m}\left[\xi_{\mathrm{I}}(x)\right]+A_{-} J_{-m}\left[\xi_{\mathrm{I}}(x)\right]\right\} \\
& :=A_{+} u_{\mathrm{I}+}(x)+A_{-} u_{\mathrm{I}-}(x) \\
u_{\mathrm{II}}(x) & =\sqrt{\frac{\xi_{\mathrm{II}}(x)}{\omega_{\mathrm{II}}(x)}}\left\{B_{+} I_{+m}\left[\xi_{\mathrm{II}}(x)\right]+B_{-} I_{-m}\left[\xi_{\mathrm{II}}(x)\right]\right\} \\
& :=B_{+} u_{\mathrm{II}+}(x)+B_{-} u_{\mathrm{II}-}(x),
\end{aligned}
$$

and the Green's functions can be written as

$$
\begin{aligned}
G_{\mathrm{I}}(x, y)= & -\frac{\pi}{\sqrt{3}} \theta(x-y) u_{\mathrm{I}-}(x) u_{\mathrm{I}+}(y) \\
& -\frac{\pi}{\sqrt{3}} \theta(y-x) u_{\mathrm{I}+}(x) u_{\mathrm{I}-}(y) \\
G_{\mathrm{II}}(x, y)= & \frac{i \pi}{\sqrt{3}} \theta(x-y) u_{\mathrm{II}-}(x) u_{\mathrm{II}+}(y) \\
& +\frac{i \pi}{\sqrt{3}} \theta(y-x) u_{\mathrm{II}+}(x) u_{\mathrm{II}-}(y) .
\end{aligned}
$$

With the ansatz

$$
\begin{gathered}
\chi_{\mathrm{I}}(x)=A_{+} a_{+}(x) u_{\mathrm{I}+}(x)+A_{-} a_{-}(x) u_{\mathrm{I}-}(x) \\
\chi_{\mathrm{II}}(x)=B_{+} b_{+}(x) u_{\mathrm{II}+}(x)+B_{-} b_{-}(x) u_{\mathrm{II}-}(x),
\end{gathered}
$$

from Eqs. (29) and (30), the problem is now reduced to the determination of the $x$-dependent coefficients

$$
\begin{aligned}
& a_{+}(x):=\left[1+\varepsilon J_{+-}^{+}\left(x, x_{i}\right)\right]+\varepsilon \frac{A_{-}}{A_{+}} J_{--}^{-}\left(x, x_{i}\right) \\
& a_{-}(x):=\left[1+\varepsilon J_{+-}^{-}\left(x_{*}, x\right)\right]+\varepsilon \frac{A_{+}}{A_{-}} J_{++}^{+}\left(x_{*}, x\right) \\
& b_{+}(x):=\left[1-i \varepsilon I_{+-}^{+}\left(x, x_{*}\right)\right]-i \varepsilon \frac{B_{-}}{B_{+}} I_{--}^{-}\left(x, x_{*}\right) \\
& b_{-}(x):=\left[1-i \varepsilon I_{+-}^{-}\left(x_{f}, x\right)\right]-i \varepsilon \frac{B_{+}}{B_{-}} I_{++}^{+}\left(x_{f}, x\right)
\end{aligned}
$$

in which, for the sake of brevity (and clarity), we have introduced a shorthand notation for the following integrals

$$
\begin{aligned}
& J_{s \bar{s}}^{w}\left(x_{1}, x_{2}\right):=\frac{\pi}{\sqrt{3}} \int_{x_{1}}^{x_{2}} \sigma_{\mathrm{I}}(y) a_{w}(y) u_{\mathrm{I} s}(y) u_{\mathrm{I} \bar{s}}(y) \mathrm{d} y \\
& =\frac{\pi}{\sqrt{3}} \int_{x_{1}}^{x_{2}} \sigma_{\mathrm{I}}(y) \frac{\xi_{\mathrm{I}}(y)}{\omega_{\mathrm{I}}(y)} a_{w}(y) J_{s m}\left[\xi_{\mathrm{I}}(y)\right] J_{\bar{s} m}\left[\xi_{\mathrm{I}}(y)\right] \mathrm{d} y \\
& I_{s \bar{s}}^{w}\left(x_{1}, x_{2}\right):=\frac{\pi}{\sqrt{3}} \int_{x_{1}}^{x_{2}} \sigma_{\mathrm{II}}(y) b_{w}(y) u_{\mathrm{II} s}(y) u_{\mathrm{II} \bar{s}}(y) \mathrm{d} y \\
& =\frac{\pi}{\sqrt{3}} \int_{x_{1}}^{x_{2}} \sigma_{\mathrm{II}}(y) \frac{\xi_{\mathrm{II}}(y)}{\omega_{\mathrm{II}}(y)} b_{w}(y) I_{s m}\left[\xi_{\mathrm{II}}(y)\right] I_{\bar{s} m}\left[\xi_{\mathrm{II}}(y)\right] \mathrm{d} y,
\end{aligned}
$$

where the symbols $w, s$ and $\bar{s}$ are + or - .

Before tackling this problem, we shall work out relations which allow us to determine the constant coefficients $A_{ \pm}$and $B_{ \pm}$uniquely.

\section{A. Initial and matching conditions}

We first use the initial conditions (4) in order to fix the constant coefficients $A_{ \pm}$.

For the function $\chi$ such conditions become

$$
\begin{aligned}
\lim _{x \rightarrow x_{i}} \chi(x) & =d \sqrt{\frac{1-\epsilon_{1}\left(x_{i}\right)}{2 k}} \mathrm{e}^{-i k \eta_{i}-x_{i} / 2} \\
& =d \sqrt{\frac{1-\epsilon_{1}\left(x_{i}\right)}{2 k}} \mathrm{e}^{i \xi_{\mathrm{I}}\left(x_{i}\right)-x_{i} / 2},
\end{aligned}
$$

where, from the definitions of $\eta$ and $x, k \eta_{i} \simeq-i \xi_{\mathrm{I}}\left(x_{i}\right)$. We then recall the asymptotic form of the Bessel functions $J_{\nu}$ for $x \rightarrow+\infty$ [27, 28] 37],

$$
J_{ \pm m}\left[\xi_{\mathrm{I}}(x)\right] \sim \sqrt{\frac{2}{\pi \xi_{\mathrm{I}}(x)}} \cos \left(\xi_{\mathrm{I}}(x) \mp \frac{\pi}{2} m-\frac{\pi}{4}\right)
$$

from which we obtain the asymptotic expression of the mode function in region $\mathrm{I}$, 


$$
\begin{array}{r}
\chi_{\mathrm{I}}\left(x_{i}\right) \sim \sqrt{\frac{1-\epsilon_{1}\left(x_{i}\right)}{2 \pi}} \mathrm{e}^{-x_{i} / 2}\left\{\mathrm{e}^{+i \xi_{\mathrm{I}}\left(x_{i}\right)}\left[\mathrm{e}^{-i\left(\frac{1}{4}+\frac{m}{2}\right) \pi} A_{+}+\mathrm{e}^{-i\left(\frac{1}{4}-\frac{m}{2}\right) \pi}\left(A_{-}+\varepsilon A_{-} J_{+-}^{-}\left(x_{*}, x_{i}\right)+\varepsilon A_{+} J_{++}^{+}\left(x_{*}, x_{i}\right)\right)\right]\right. \\
\left.+\mathrm{e}^{-i \xi_{\mathrm{I}}\left(x_{i}\right)}\left[\mathrm{e}^{+i\left(\frac{1}{4}+\frac{m}{2}\right) \pi} A_{+}+\mathrm{e}^{+i\left(\frac{1}{4}-\frac{m}{2}\right) \pi}\left(A_{-}+\varepsilon A_{-} J_{+-}^{-}\left(x_{*}, x_{i}\right)+\varepsilon A_{+} J_{++}^{+}\left(x_{*}, x_{i}\right)\right)\right]\right\}
\end{array}
$$

The initial conditions (34) therefore yield

$$
\begin{aligned}
& A_{+}=d \sqrt{\frac{\pi}{k}} \frac{\mathrm{e}^{+i\left(\frac{1}{4}+\frac{m}{2}\right) \pi}}{1-\mathrm{e}^{2 i m \pi}} \\
& A_{-}=-A_{+} \frac{\mathrm{e}^{+i m \pi}+\varepsilon J_{++}^{+}\left(x_{*}, x_{i}\right)}{1+\varepsilon J_{+-}^{-}\left(x_{*}, x_{i}\right)} .
\end{aligned}
$$

Note that $A_{+}$does not depend on $\varepsilon$, therefore the result (37a holds to all orders.

We next impose continuity at the turning point in order to determine the coefficients $B_{ \pm}$. For this we shall need the (asymptotic) expressions of Bessel functions for $x \rightarrow x_{*}$ [27, 28] [38]. In particular,

$$
\begin{aligned}
& J_{ \pm m}\left[\xi_{\mathrm{I}}(x)\right] \simeq\left[\frac{\xi_{\mathrm{I}}(x)}{2}\right]^{ \pm m} \frac{1}{\Gamma(1 \pm m)} \text { for } x \rightarrow x_{*}^{+} \\
& I_{ \pm m}\left[\xi_{\mathrm{II}}(x)\right] \simeq\left[\frac{\xi_{\mathrm{II}}(x)}{2}\right]^{ \pm m} \frac{1}{\Gamma(1 \pm m)} \text { for } x \rightarrow x_{*}^{-},
\end{aligned}
$$

where $\Gamma$ is Euler's gamma function. Near the turning point, we just keep the leading term in the expansion (23), so that

$$
\begin{aligned}
& \omega(x) \simeq \sqrt{C}\left|x-x_{*}\right|^{\frac{1-2 m}{2 m}} \\
& \xi(x) \simeq 2 m \sqrt{C}\left|x-x_{*}\right|^{\frac{1}{2 m}} .
\end{aligned}
$$

We thus obtain the following forms of the mode functions near the turning point,

$$
\begin{aligned}
\chi_{\mathrm{I}}\left(x \simeq x_{*}\right) \simeq & A_{+} a_{+}\left(x_{*}\right) \frac{\sqrt{2 m}(m \sqrt{C})^{m}}{\Gamma(1+m)}\left(x-x_{*}\right) \\
& +A_{-} a_{-}\left(x_{*}\right) \frac{\sqrt{2 m}(m \sqrt{C})^{-m}}{\Gamma(1-m)} \\
\chi_{\mathrm{II}}\left(x \simeq x_{*}\right) \simeq & B_{+} b_{+}\left(x_{*}\right) \frac{\sqrt{2 m}(m \sqrt{C})^{m}}{\Gamma(1+m)}\left(x_{*}-x\right) \\
& +B_{-} b_{-}\left(x_{*}\right) \frac{\sqrt{2 m}(m \sqrt{C})^{-m}}{\Gamma(1-m)} .
\end{aligned}
$$

Continuity across $x_{*}$ then implies that

$$
\begin{aligned}
& B_{+} b_{+}\left(x_{*}\right)=-A_{+} a_{+}\left(x_{*}\right) \\
& B_{-} b_{-}\left(x_{*}\right)=A_{-} a_{-}\left(x_{*}\right) .
\end{aligned}
$$

From the definitions (32) at $x=x_{*}$, Eq. (37b) and the relations (41) we therefore obtain

$$
\begin{aligned}
\frac{B_{+}}{A_{+}}= & -1+\varepsilon \frac{\mathrm{e}^{+i m \pi}+\varepsilon J_{++}^{+}\left(x_{*}, x_{i}\right)}{1+\varepsilon J_{+-}^{-}\left(x_{*}, x_{i}\right)} J_{--}^{-}\left(x_{*}, x_{i}\right) \\
& -\varepsilon J_{+-}^{+}\left(x_{*}, x_{i}\right) \\
\frac{B_{-}}{A_{+}}= & -\frac{\mathrm{e}^{+i m \pi}+\varepsilon J_{++}^{+}\left(x_{*}, x_{i}\right)}{1+\varepsilon J_{+-}^{-}\left(x_{*}, x_{i}\right)} \\
& \times \frac{1-i \varepsilon^{2} J_{--}^{-}\left(x_{*}, x_{i}\right) I_{++}^{+}\left(x_{f}, x_{*}\right)}{1-i \varepsilon I_{+-}^{-}\left(x_{f}, x_{*}\right)} \\
& -i \varepsilon \frac{1+\varepsilon J_{+-}^{+}\left(x_{*}, x_{i}\right)}{1-i \varepsilon I_{+-}^{-}\left(x_{f}, x_{*}\right)} I_{++}^{+}\left(x_{f}, x_{*}\right) .
\end{aligned}
$$

It is apparent from the above derivation that, since the matching between approximate solutions in the two regions is always performed at $x=x_{*}$, no ambiguity hinders the evaluation of the coefficients $B_{ \pm}$and the accuracy of the approximate solution is therefore expected to increase with increasing order.

\section{B. Recursive (perturbative) relations}

We can finally obtain expressions for the coefficients $a_{ \pm}(x)$ and $b_{ \pm}(x)$ by solving the integral relations (32). We remark that the r.h.s.'s of such equations contain $a_{ \pm}(x)$ and $b_{ \pm}(x)$, which can therefore be determined recursively by expanding them (as well as any function of them) in $\varepsilon$, that is

$$
\begin{aligned}
& a_{ \pm}(x)=1+\sum_{q \geq 1} \varepsilon^{q} a_{ \pm}^{(q)} \\
& b_{ \pm}(x)=1+\sum_{q \geq 1} \varepsilon^{q} b_{ \pm}^{(q)} .
\end{aligned}
$$

It will also be useful to define the following integrals

$$
\begin{aligned}
& J_{s \bar{s}}^{w(q)}\left(x_{1}, x_{2}\right):=\frac{\pi}{\sqrt{3}} \int_{x_{1}}^{x_{2}} \sigma_{\mathrm{I}}(y) a_{w}^{(q)}(y) u_{I s}(y) u_{I \bar{s}}(y) \mathrm{d} y \\
& I_{s \bar{s}}^{w(q)}\left(x_{1}, x_{2}\right):=\frac{\pi}{\sqrt{3}} \int_{x_{1}}^{x_{2}} \sigma_{\mathrm{II}}(y) b_{w}^{(q)}(y) u_{I I s}(y) u_{I I \bar{s}}(y) \mathrm{d} y,
\end{aligned}
$$

where again $w, s$ and $\bar{s}= \pm$, and $q$ is a non-negative integer. 


\section{Leading order}

We begin by considering the leading $\left(\varepsilon^{0}\right)$ order

$$
a_{ \pm}^{(0)}(x)=b_{ \pm}^{(0)}(x)=1 .
$$

The corresponding solutions are simply given by

$$
\begin{aligned}
& \chi_{\mathrm{I}}(x) \simeq u_{\mathrm{I}}(x) \\
& =A_{+} \sqrt{\frac{\xi_{\mathrm{I}}(x)}{\omega_{\mathrm{I}}(x)}}\left\{J_{+m}\left[\xi_{\mathrm{I}}(x)\right]-\mathrm{e}^{+i m \pi} J_{-m}\left[\xi_{\mathrm{I}}(x)\right]\right\} \\
& \chi_{\mathrm{II}}(x) \simeq u_{\mathrm{II}}(x) \\
& =-A_{+} \sqrt{\frac{\xi_{\mathrm{II}}(x)}{\omega_{\mathrm{II}}(x)}}\left\{I_{+m}\left[\xi_{\mathrm{II}}(x)\right]+\mathrm{e}^{+i m \pi} I_{-m}\left[\xi_{\mathrm{II}}(x)\right]\right\},
\end{aligned}
$$

where we used the zero order forms of Eqs. 37b and (42),

$$
\begin{aligned}
& A_{-}=B_{-}=-A_{+} \mathrm{e}^{+i m \pi} \\
& B_{+}=-A_{+},
\end{aligned}
$$

and $A_{+}$is given in Eq. (37a).

We know that these expressions are also good solutions of Eq. (10) near the turning point and, deep in region II, we can use the asymptotic form of $I_{\nu}$ for $x \sim x_{f} \rightarrow$ $-\infty$ [27, 28],

$$
\begin{aligned}
I_{ \pm m}\left[\xi_{\mathrm{II}}(x)\right] \sim & \frac{1}{\sqrt{2 \pi \xi_{\mathrm{II}}(x)}} \\
& \times\left[\mathrm{e}^{\xi_{\mathrm{II}}(x)}+\mathrm{e}^{-\xi_{\mathrm{II}}(x)-i\left(\frac{1}{2} \pm m\right) \pi}\right] .
\end{aligned}
$$

On neglecting the non-leading mode $\mathrm{e}^{-\xi_{\text {II }}(x)}$, we then obtain

$$
\chi_{\mathrm{II}}\left(x_{f}\right) \sim-A_{+} \frac{\left(1+\mathrm{e}^{+i m \pi}\right)}{\sqrt{2 \pi \omega_{\mathrm{II}}\left(x_{f}\right)}} \mathrm{e}^{\xi_{\mathrm{II}}\left(x_{f}\right)} .
$$

If we now use Eqs. (5a), (37a and (49), and the values of $d$, we recover the results of Ref. [8] for all quantities of interest, i.e. power spectra, spectral indices and $\alpha$ runnings

$$
\begin{aligned}
\mathcal{P}_{\zeta} & \simeq \frac{H^{2}}{\pi \epsilon_{1} m_{\mathrm{PI}}^{2}}\left(\frac{k}{a H}\right)^{3} \frac{\mathrm{e}^{2 \xi_{\mathrm{II}, \mathrm{S}}(k, \eta)}}{\left[1-\epsilon_{1}(\eta)\right] \omega_{\mathrm{II}, \mathrm{S}}(k, \eta)} \\
& :=\mathcal{P}_{\zeta}^{(0)} \\
n_{\mathrm{S}} & -1 \simeq 3+\left.2 \frac{\mathrm{d} \xi_{\mathrm{II}, \mathrm{S}}}{\mathrm{d} \ln k}\right|_{k=k_{*}} \\
\alpha_{\mathrm{S}} & \left.\simeq 2 \frac{\mathrm{d}^{2} \xi_{\mathrm{II}, \mathrm{S}}}{(\mathrm{d} \ln k)^{2}}\right|_{k=k_{*}},
\end{aligned}
$$

and

$$
\begin{aligned}
\mathcal{P}_{h} & \simeq \frac{16 H^{2}}{\pi m_{\mathrm{Pl}}^{2}}\left(\frac{k}{a H}\right)^{3} \frac{\mathrm{e}^{2 \xi_{\mathrm{II}, \mathrm{T}}(k, \eta)}}{\left[1-\epsilon_{1}(\eta)\right] \omega_{\mathrm{II}, \mathrm{T}}(k, \eta)} \\
& :=\mathcal{P}_{h}^{(0)} \\
n_{\mathrm{T}} & \simeq 3+\left.2 \frac{\mathrm{d} \xi_{\mathrm{II}, \mathrm{T}}}{\mathrm{d} \ln k}\right|_{k=k_{*}} \\
\alpha_{\mathrm{T}} & \left.\simeq 2 \frac{\mathrm{d}^{2} \xi_{\mathrm{II}, \mathrm{T}}}{(\mathrm{d} \ln k)^{2}}\right|_{k=k_{*}},
\end{aligned}
$$

where we have transformed back to the original variables $k$ and $\eta$, and all quantities are evaluated in the superhorizon limit (i.e. for $k \ll a H$ ).

\section{Next-to-leading order}

We now insert the leading order expressions (45) into the integral relations (32) to compute the next-to-leading order expressions

$$
\begin{aligned}
& a_{+}^{(1)}(x)=J_{+-}^{(0)}\left(x, x_{i}\right)-\mathrm{e}^{+i m \pi} J_{--}^{(0)}\left(x, x_{i}\right) \\
& a_{-}^{(1)}(x)=J_{+-}^{(0)}\left(x_{*}, x\right)-\mathrm{e}^{-i m \pi} J_{++}^{(0)}\left(x_{*}, x\right) \\
& b_{+}^{(1)}(x)=-i I_{+-}^{(0)}\left(x, x_{*}\right)-i \mathrm{e}^{+i m \pi} I_{--}^{(0)}\left(x, x_{*}\right) \\
& b_{-}^{(1)}(x)=-i I_{+-}^{(0)}\left(x_{f}, x\right)-i \mathrm{e}^{-i m \pi} I_{++}^{(0)}\left(x_{f}, x\right),
\end{aligned}
$$

where we used the simplified notation $J_{s \bar{s}}^{+(0)}=J_{s \bar{s}}^{-(0)}=$ : $J_{s \bar{s}}^{(0)}$ and $I_{s \bar{s}}^{+(0)}=I_{s \bar{s}}^{-(0)}=: I_{s \bar{s}}^{(0)}$, and also expanded the coefficients $A_{-}$and $B_{ \pm}$to zero order in $\varepsilon$.

From Eq. (48), again neglecting the non-leading mode $\mathrm{e}^{-\xi_{\mathrm{II}}(x)}$, we obtain the mode function at $x=x_{f}$ to first order in $\varepsilon$,

$$
\begin{aligned}
\chi_{\mathrm{II}}\left(x_{f}\right) \sim- & \frac{A_{+} \mathrm{e}^{\xi_{\mathrm{II}}\left(x_{f}\right)}}{\sqrt{2 \pi \omega_{\mathrm{II}}\left(x_{f}\right)}}\left\{1+\mathrm{e}^{+i m \pi}\right. \\
& +i \varepsilon\left[I_{++}^{(0)}\left(x_{f}, x_{*}\right)-I_{+-}^{(0)}\left(x_{f}, x_{*}\right)\right] \\
& -i \varepsilon \mathrm{e}^{+i m \pi}\left[I_{--}^{(0)}\left(x_{f}, x_{*}\right)-I_{+-}^{(0)}\left(x_{f}, x_{*}\right)\right] \\
& -\varepsilon \mathrm{e}^{+i m \pi}\left[J_{+-}^{(0)}\left(x_{*}, x_{i}\right)+J_{--}^{(0)}\left(x_{*}, x_{i}\right)\right] \\
& \left.+\varepsilon\left[J_{+-}^{(0)}\left(x_{*}, x_{i}\right)+J_{++}^{(0)}\left(x_{*}, x_{i}\right)\right]\right\},
\end{aligned}
$$

which, using Eqs. (5a), (37a), (52) and the values of $d$, yields all quantities of interest to first order in $\varepsilon$. For the spectra we obtain

$$
\begin{aligned}
& \mathcal{P}_{\zeta} \simeq \mathcal{P}_{\zeta}^{(0)}\left[1+g_{(1) \mathrm{S}}^{\mathrm{GREEN}}\left(x_{f}\right)\right] \\
& \mathcal{P}_{h} \simeq \mathcal{P}_{h}^{(0)}\left[1+g_{(1) \mathrm{T}}^{\mathrm{GREEN}}\left(x_{f}\right)\right],
\end{aligned}
$$


where the relative corrections to the leading order expressions (50a) and (50d) are now given by

$$
\begin{aligned}
& g_{(1) \mathrm{S}, \mathrm{T}}^{\mathrm{GREEN}}(x)=\left\{J_{++}^{(0)}\left(x_{*}, x_{i}\right)-J_{--}^{(0)}\left(x_{*}, x_{i}\right)\right. \\
& \left.+\frac{1}{\sqrt{3}}\left[I_{++}^{(0)}\left(x, x_{*}\right)-2 I_{+-}^{(0)}\left(x, x_{*}\right)+I_{--}^{(0)}\left(x, x_{*}\right)\right]\right\}_{\mathrm{S}, \mathrm{T}},
\end{aligned}
$$

in which we set $\varepsilon=1$ and $n=1$ as required, and write $\mathrm{S}$ and $\mathrm{T}$ to recall the use of the corresponding frequencies. The expressions for the spectral indices and their runnings to this order can finally be derived from the definitions (5b) and (5c).

\section{Higher orders}

The above procedure can be extended to all orders. The coefficients up to $a_{ \pm}^{(q)}$ and $b_{ \pm}^{(q)}$ must be used to compute the integrals $J_{s \bar{s}}^{w(q)}$ and $I_{s \bar{s}}^{w(q)}$ which determine $a_{ \pm}^{(q+1)}$ and $b_{ \pm}^{(q+1)}$. However, general expressions soon become very involved, and we shall test the effectiveness of our method by applying it to a case of interest in Section VI

\section{ADIABATIC EXPANSION}

Let us now apply the usual adiabatic expansion with the assumption that the leading order be given by the functions (19) [rather than the more common expression [12] ]. This leads one to replace

$$
\xi(x) \rightarrow \frac{\xi(x)}{\sqrt{\delta}}:=\frac{1}{\sqrt{\delta}} \int^{x} \omega(y) \mathrm{d} y,
$$

and consider the forms $[23]$.

$$
U_{ \pm}=F_{ \pm}(x ; \delta) u_{ \pm}(x)+G_{ \pm}(x ; \delta) u_{ \pm}^{\prime}(x),
$$

where the $x$-dependent coefficients $F$ and $G$ must now be determined. The particular case of a linear turning point 29] (precisely the one which mostly concerns us here), can then be fully analyzed as follows.

\section{A. Recursive (adiabatic) relations}

The $x$-dependent coefficients $F$ and $G$ can be expanded in powers of $\delta$ as

$$
\begin{aligned}
& F(x ; \delta)=\sum_{j \geq 0} \delta^{j} \phi_{(j)}(x) \\
& G(x ; \delta)=\sum_{j \geq 0} \delta^{j} \gamma_{(j)}(x) .
\end{aligned}
$$

Upon substituting into Eq. (25) with $\varepsilon=1$, one therefore obtains that the coefficients $\phi_{(j)}$ and $\gamma_{(j)}$ are given recursively by the formulae

$$
\begin{aligned}
\phi_{(j)}(x)=-\frac{1}{2} \int^{x}\left[\gamma_{(j)}^{\prime \prime}(y)+\sigma(y) \gamma_{(j)}(y)\right] \mathrm{d} y \\
\gamma_{(j)}(x)=\frac{1}{2 \omega(x)} \int^{x}\left\{\sigma(y)\left[2 \gamma_{(j-1)}^{\prime}(y)+\phi_{(j-1)}(y)\right]\right. \\
\left.+\sigma^{\prime}(y) \gamma_{(j-1)}(y)+\phi_{(j-1)}^{\prime \prime}(y)\right\} \frac{\mathrm{d} y}{\omega(y)},
\end{aligned}
$$

where it is understood that the integration must be performed from $x_{*}$ to $x$ in region I and from $x$ to $x_{*}$ in region II. Let us also remark that, for $\omega^{2} \sim\left(x-x_{*}\right)$ near the turning point, the above expressions are finite [29], whereas for more general cases one expects divergences as with the more standard WKB approach.

\section{Leading order}

The lowest order solutions (19) are correctly recovered on setting

$$
\phi_{(0)}=1 \text { and } \gamma_{(0)}=0,
$$

in the limit $\delta=1$. The relevant leading order perturbations are therefore obtained on imposing the initial conditions (34) and matching conditions between region I and region II at the turning point, which therefore yield for $\chi_{\mathrm{I}}$ and $\chi_{\mathrm{II}}$ the same linear combinations $u_{\mathrm{I}}$ of Eq. 46a and $u_{\mathrm{II}}$ of Eq. (46b) previously obtained.

\section{Higher orders}

The condition (56) makes the formal expressions of the coefficients $\phi_{(1)}$ and $\gamma_{(1)}$ particularly simple,

$$
\begin{aligned}
& \phi_{(1)}(x)=-\frac{1}{2} \int^{x}\left[\gamma_{(1)}^{\prime \prime}(y)+\sigma(y) \gamma_{(1)}(y)\right] \mathrm{d} y \\
& \gamma_{(1)}(x)=\frac{1}{2 \omega(x)} \int^{x} \frac{\sigma(y)}{\omega(y)} \mathrm{d} y,
\end{aligned}
$$

and the first order solutions are then given by linear combinations of the two functions

$$
U_{ \pm}(x)=\left[1+\delta \phi_{(1)}(x)\right] u_{ \pm}(x)+\delta \gamma_{(1)}(x) u_{ \pm}^{\prime}(x) .
$$

It is now important to observe that the initial conditions (34) and matching conditions at the turning point to first order in the adiabatic parameter $\delta$ yield different results with respect to the perturbative expansion in $\varepsilon$. First of all, the coefficient $A_{+}$, which was left unaffected by the expansion in $\varepsilon$ [see Eq. [37a ], acquires a correction. The relations between $A_{-}, B_{ \pm}$and $A_{+}$instead remain those given in Eq. 477) to all orders, whereas in the perturbative expansion they were modified [see 
Eqs. (37b) and [42)]. To summarize, to first order in $\delta$, one obtains

$$
\begin{aligned}
A_{+} \simeq & d \sqrt{\frac{\pi}{k}} \frac{\mathrm{e}^{i\left(\frac{1}{4}+\frac{m}{2}\right) \pi}}{1-\mathrm{e}^{2 i m \pi}} \\
& \times\left\{1-\delta\left[\phi_{\mathrm{I}(1)}\left(x_{i}\right)+\gamma_{\mathrm{I}(1)}\left(x_{i}\right)\left(i \omega_{\mathrm{I}}\left(x_{i}\right)-\frac{1}{2}\right)\right]\right\} \\
A_{-}= & -A_{+} \mathrm{e}^{+i m \pi} \\
B_{ \pm}= & \mp A_{ \pm}
\end{aligned}
$$

and the perturbation modes deep in Region II (at $x \ll$ $x_{*}$ ) are finally given by

$$
\chi_{\mathrm{II}}(x) \simeq u_{\mathrm{II}}(x)\left\{1+\delta\left[\phi_{\mathrm{II}(1)}(x)-\gamma_{\mathrm{II}(1)}(x)\left(\omega_{\mathrm{II}}(x)+\frac{\omega_{\mathrm{II}}^{\prime}(x)}{2 \omega_{\mathrm{II}}(x)}\right)-\phi_{\mathrm{I}(1)}\left(x_{i}\right)+\gamma_{\mathrm{I}(1)}\left(x_{i}\right)\left(\frac{1}{2}-i \omega_{\mathrm{I}}\left(x_{i}\right)\right)\right]\right\}
$$

We note that, in order to obtain the above result, one must first take the asymptotic expansion of the functions $u_{ \pm}$and then take the derivative, since the reverse order would lead to larger errors. The corrected power spectra are then given by

$$
\begin{aligned}
& \mathcal{P}_{\zeta} \simeq \mathcal{P}_{\zeta}^{(0)}\left[1+g_{(1) \mathrm{S}}^{\mathrm{AD}}\left(x_{f}\right)\right] \\
& \mathcal{P}_{h} \simeq \mathcal{P}_{h}^{(0)}\left[1+g_{(1) \mathrm{T}}^{\mathrm{AD}}\left(x_{f}\right)\right],
\end{aligned}
$$

in which we set $\delta=1$ at the end, and 39

$$
\begin{aligned}
g_{(1) \mathrm{S}, \mathrm{T}}^{\mathrm{AD}}(x)=2[ & \phi_{\mathrm{II}(1)}(x)-\gamma_{\mathrm{II}(1)}(x)\left(\omega_{\mathrm{II}}(x)+\frac{\omega_{\mathrm{II}}^{\prime}(x)}{2 \omega_{\mathrm{II}}(x)}\right) \\
& \left.+\frac{\gamma_{\mathrm{I}(1)}\left(x_{i}\right)}{2}-\phi_{\mathrm{I}(1)}\left(x_{i}\right)\right]_{\mathrm{S}, \mathrm{T}},
\end{aligned}
$$

where the indices $\mathrm{S}$ and $\mathrm{T}$ recall the use of the corresponding frequencies. As usual, the corrections for the spectral indices and $\alpha$-runnings then follow from their definitions (5b) and (5c).

Given the complicated expressions for $\omega$, it is usually impossible to carry out the double integration that yields $\phi_{(1)}$ analytically, let alone higher order terms. One must therefore rely on numerical computations. In Section VI we shall, in the context of power-law inflation, compare the results obtained in next-to-leading order from the two expansions so far described in full generality.

\section{APPLICATION TO POWER-LAW INFLATION}

In this model the scale factor is given by

$$
a(\eta)=\ell_{0}|\eta|^{1+\beta},
$$

with $\beta \leq-2$. The case $\beta=-2$ is special, since it corresponds to the de Sitter space-time with constant Hubble radius equal to $\ell_{0}$. The frequency is given, both for scalar and tensor modes, by

$$
\omega^{2}(x)=(1+\beta)^{2} \mathrm{e}^{2 x}-\left(\beta+\frac{1}{2}\right)^{2},
$$

and the horizon flow functions read

$$
\epsilon_{1}=\frac{2+\beta}{1+\beta}, \quad \epsilon_{n}=0, \quad n>1
$$

For the particular case $\beta=-2$, we plot the frequency in Fig. 1 the function $\xi(x)$ in Fig. 2 and the perturbation $\sigma(x)$ in Fig. 3 .

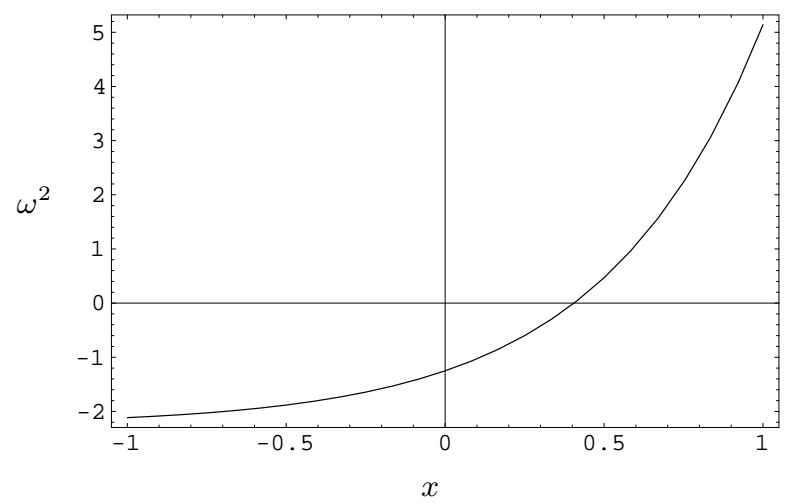

FIG. 1: The frequency $\omega^{2}$ for $\beta=-2$. The turning point is at $x_{*}=\ln (3 / 2) \simeq 0.4$

Eq. (2) with the initial conditions (4) can be solved analytically and the exact power spectra at $x_{f} \rightarrow-\infty$ 


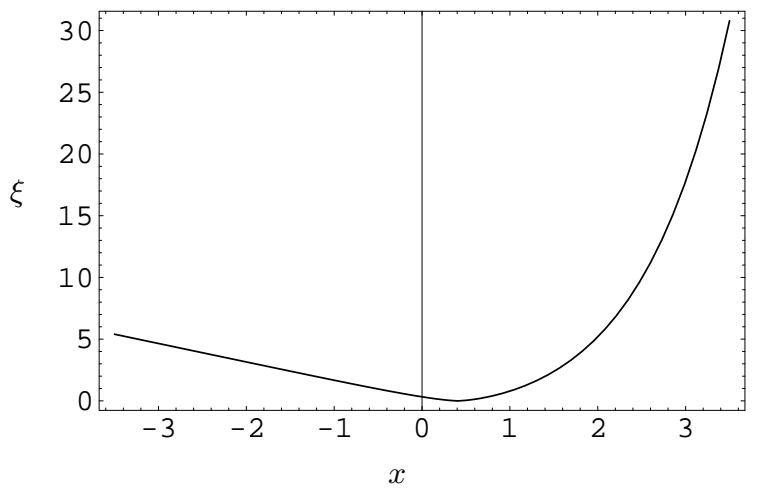

FIG. 2: The function $\xi$ for $\beta=-2$.

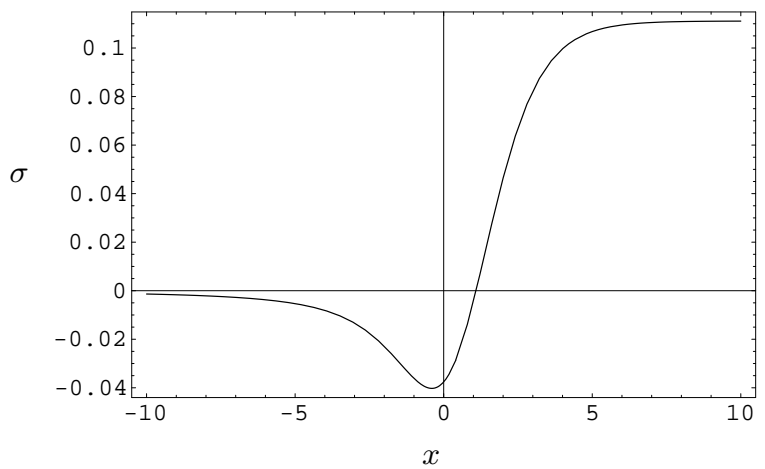

FIG. 3: The "perturbation" $\sigma$ for $\beta=-2$.

are given by (see, e.g. Refs. [5, [8, 30, 31])

$$
\begin{aligned}
& \mathcal{P}_{\zeta}=\frac{1}{\pi \epsilon_{1} m_{\mathrm{Pl}}^{2}} \frac{1}{l_{0}^{2}} f(\beta) k^{2 \beta+4} \\
& \mathcal{P}_{h}=\frac{16}{\pi m_{\mathrm{Pl}}^{2}} \frac{1}{l_{0}^{2}} f(\beta) k^{2 \beta+4},
\end{aligned}
$$

where

$$
f(\beta):=\frac{1}{\pi}\left[\frac{\Gamma(|\beta+1 / 2|)}{2^{\beta+1}}\right]^{2},
$$

and $f(\beta=-2)=1[40]$. The spectral indices and their runnings can also be calculated from Eqs. (5b) and (5c) and one finds $n_{\mathrm{S}}-1=n_{\mathrm{T}}=2 \beta+4$ and $\alpha_{\mathrm{S}}=\alpha_{\mathrm{T}}=0$. Exact scale-invariance is obtained for $\beta=-2$.

\section{A. Leading order results}

The leading order expressions in the perturbative expansion of Section [V] as well as the adiabatic expansion of Section $\square$ lead to the same result as in Ref. [8],

$$
\begin{aligned}
& \mathcal{P}_{\zeta} \simeq \frac{1}{\pi \epsilon_{1} m_{\mathrm{Pl}}^{2}} \frac{1}{l_{0}^{2}} g^{(0)}(\beta) k^{2 \beta+4} \\
& \mathcal{P}_{h} \simeq \frac{16}{\pi m_{\mathrm{Pl}}^{2}} \frac{1}{l_{0}^{2}} g^{(0)}(\beta) k^{2 \beta+4},
\end{aligned}
$$

where the function $g^{(0)}(\beta)$ is given by

$$
g^{(0)}(\beta):=\frac{2 e^{2 \beta+1}}{|2 \beta+1|^{2 \beta+2}} .
$$

This yields a relative error for the amplitude of the power spectrum

$$
\Delta_{P}^{(0)}:=100\left|\frac{f(\beta)-g^{(0)}(\beta)}{f(\beta)}\right| \%,
$$

which decreases for increasing $|\beta|$, as can be seen from Fig. 4 but is rather large (about 10\%) for the de Sitter space-time. The spectral indices and their runnings are instead predicted exactly as $n_{\mathrm{S}}-1=n_{\mathrm{T}}=2 \beta+4$ and $\alpha_{\mathrm{S}}=\alpha_{\mathrm{T}}=0$.

\section{B. Next-to-leading order results}

We shall now compare the corrections to the amplitude of the power spectra coming from the two different expansions we described in Section

\section{Perturbative expansion}

From the next-to-leading expressions in SectionIVB2 we obtain that the relative correction to the power spectra is given by the function $g_{(1)}^{\text {GREEN }}:=g_{(1) \mathrm{S}}^{\text {GREEN }}=$ $g_{(1) \mathrm{T}}^{\text {GREEN }}$ in Eq. (53b) evaluated at $x=x_{f} \ll x_{*}$. This quantity can be determined numerically and one finds that it approaches an asymptotical finite value for $x_{f} \rightarrow$ $-\infty$. Some examples are given in Table $\square$ for $x_{f}=-13$, from which it is clear that the improvement over the leading order is small. Once the correction has been included, the total relative error on the power spectra (denoted by $\left.\Delta_{P}^{(1)}\right)$ therefore remains of the same order as the leading order error $\Delta_{P}^{(0)}$.

\section{Adiabatic expansion}

We analogously evaluate the coefficients $\phi_{(1)}$ and $\gamma_{(1)}$ numerically. For the case $\beta=-2$, the result is plotted in Fig. 15 from which it appears that the functions $\phi_{(1)}(x)$ and $\gamma_{(1)}(x)$ converge to finite asymptotic values for $x \rightarrow \pm \infty$. Since the same behavior is also found for all $\beta<-2$, we exhibit the values of such coefficients 


\begin{tabular}{|c|c|c|c|c|c|c|c|c|c|}
\hline$\beta$ & -2 & -3 & -4 & -5 & -6 & -7 & -8 & -9 & -10 \\
\hline$g_{(1)}^{\text {GREEN }}$ & $+9.6 \cdot 10^{-5}$ & $+7.3 \cdot 10^{-4}$ & $+7.1 \cdot 10^{-4}$ & $+6.4 \cdot 10^{-4}$ & $+5.8 \cdot 10^{-4}$ & $+5.2 \cdot 10^{-4}$ & $+4.8 \cdot 10^{-4}$ & $+4.4 \cdot 10^{-4}$ & $+4.2 \cdot 10^{-4}$ \\
\hline$\Delta_{P}^{(0)}$ & $10.4 \%$ & $6.4 \%$ & $4.6 \%$ & $3.6 \%$ & $3.0 \%$ & $2.5 \%$ & $2.2 \%$ & $1.9 \%$ & $1.7 \%$ \\
\hline$\Delta_{P}^{(1)}$ & $10.4 \%$ & $6.3 \%$ & $4.6 \%$ & $3.6 \%$ & $2.9 \%$ & $2.5 \%$ & $2.1 \%$ & $1.9 \%$ & $1.7 \%$ \\
\hline
\end{tabular}

TABLE I: Next-to-leading order improvement for the power spectra $\left(g_{(1)}^{\text {GREEN }}\right)$ and total final error $\left(\Delta_{P}^{(1)}\right)$ to first order in $\varepsilon$. The latter is essentially the same as to leading order $\left(\Delta_{P}^{(0)}\right)$.

\begin{tabular}{|c|c|c|c|c|c|c|c|c|c|}
\hline$\beta$ & -2 & -3 & -4 & -5 & -6 & -7 & -8 & -9 & -10 \\
\hline$\phi_{\mathrm{I}(1)}$ & $+3.2 \cdot 10^{-3}$ & $+1.1 \cdot 10^{-3}$ & $+6.0 \cdot 10^{-4}$ & $+3.6 \cdot 10^{-4}$ & $+2.4 \cdot 10^{-4}$ & $+1.7 \cdot 10^{-4}$ & $+1.3 \cdot 10^{-4}$ & $+1.0 \cdot 10^{-4}$ & $+8.1 \cdot 10^{-5}$ \\
\hline$\gamma_{\mathrm{I}(1)}$ & $-2.4 \cdot 10^{-19}$ & $-5.9 \cdot 10^{-20}$ & $-2.6 \cdot 10^{-20}$ & $-1.5 \cdot 10^{-20}$ & $-9.4 \cdot 10^{-21}$ & $-6.6 \cdot 10^{-21}$ & $-4.8 \cdot 10^{-21}$ & $-3.7 \cdot 10^{-21}$ & $-2.9 \cdot 10^{-21}$ \\
\hline$\phi_{\mathrm{II}(1)}$ & $+4.5 \cdot 10^{-3}$ & $+1.6 \cdot 10^{-3}$ & $+8.2 \cdot 10^{-4}$ & $+5.0 \cdot 10^{-4}$ & $+3.3 \cdot 10^{-4}$ & $+2.4 \cdot 10^{-4}$ & $+1.8 \cdot 10^{-4}$ & $+1.4 \cdot 10^{-4}$ & $+1.1 \cdot 10^{-4}$ \\
\hline$\gamma_{\mathrm{II}(1)}$ & $-3.5 \cdot 10^{-2}$ & $-1.2 \cdot 10^{-2}$ & $-6.4 \cdot 10^{-3}$ & $-3.8 \cdot 10^{-3}$ & $-2.6 \cdot 10^{-3}$ & $-1.8 \cdot 10^{-3}$ & $-1.4 \cdot 10^{-3}$ & $-1.1 \cdot 10^{-3}$ & $-8.6 \cdot 10^{-4}$ \\
\hline$g_{(1)}^{\mathrm{AD}}$ & $+1.1 \cdot 10^{-1}$ & $+6.3 \cdot 10^{-2}$ & $+4.5 \cdot 10^{-2}$ & $+3.5 \cdot 10^{-2}$ & $+2.9 \cdot 10^{-2}$ & $+2.4 \cdot 10^{-2}$ & $+2.1 \cdot 10^{-2}$ & $+1.8 \cdot 10^{-2}$ & $+1.6 \cdot 10^{-2}$ \\
\hline$\Delta_{P}^{(1)}$ & $0.83 \%$ & $0.50 \%$ & $0.35 \%$ & $0.27 \%$ & $0.22 \%$ & $0.18 \%$ & $0.16 \%$ & $0.14 \%$ & $0.12 \%$ \\
\hline
\end{tabular}

TABLE II: First order coefficients $\phi_{(1)}$ and $\gamma_{(1)}$, correction $\left(g_{(1)}^{\mathrm{AD}}\right)$ and total relative error $\left(\Delta_{P}^{(1)}\right)$ for the power spectrum to first order in $\delta$.

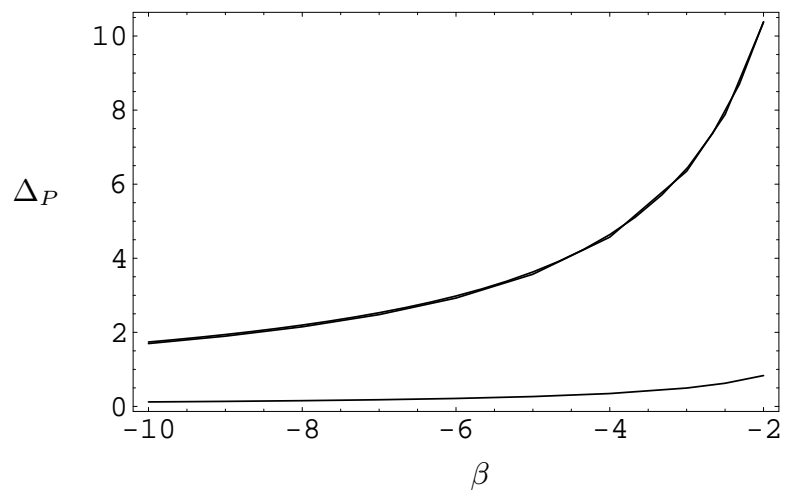

FIG. 4: The total error on the power spectrum to leading order and to next-to-leading order for the perturbative correction (superposed upper lines) and to next-to-leading order for the adiabatic correction (lower line).

(with $x_{i}=20$ and $x_{f}=-13$ ) in Table $\llbracket$ together with the corresponding next-to-leading order relative improvement $\left[g_{(1)}^{\mathrm{AD}}\left(x_{f}\right)\right.$ as defined in Eq. [61b) $]$ and the total error on the power spectra $\left(\Delta_{P}^{(1)}\right)$. On comparing with the last row in Table I] we can therefore conclude that the adiabatic corrections are significantly better than those obtained from the perturbative expansion and, in fact, yield the amplitudes with extremely high accuracy (see also Fig. (4). In fact, this method seems (at least) as accurate as the next-to-leading order in the uniform approximation employed in Ref. [16].

\section{CONCLUSIONS}

In this paper we have improved the WKB approximation for the purpose of estimating the spectra of cosmo-

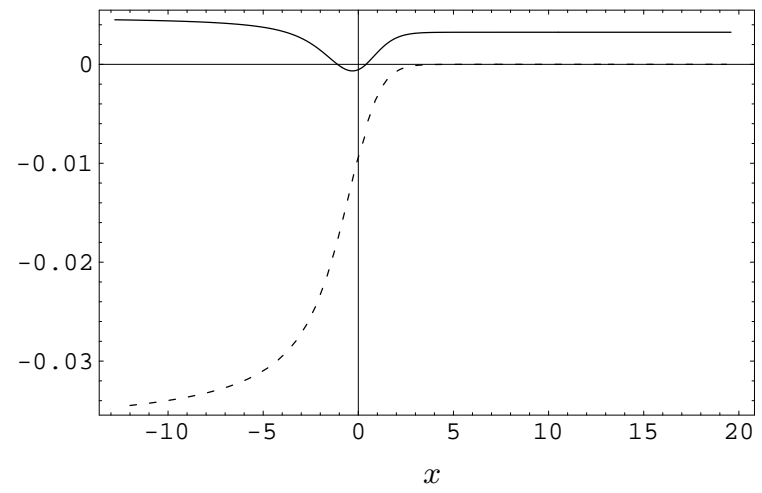

FIG. 5: The functions $\phi_{(1)}(x)$ (solid line) and $\gamma_{(1)}(x)$ (dotted line) for $\beta=-2$.

logical perturbations during inflation. We found general formulae for amplitudes, spectral indices and $\alpha$-runnings of the fluctuations to next-to-leading order both in the adiabatic expansion of Ref. 23] and a new perturbative expansion which makes use of the Green's function technique.

We have then applied our method to power-law inflation in order to test it against exact results. It is known that the spectral indices and their runnings are obtained exactly in leading order, hence we focussed on the spectra to next-to-leading order. We have found that the perturbative corrections remain too small to yield any significant improvement, whereas the adiabatic expansion to next-to-leading order reproduces the exact amplitudes with great accuracy (see Ref. 16] for a similar result with the uniform approximation). This result does not however mean that the perturbative expansion will not lead to significant corrections in different inflationary scenarios.

One way of understanding the difference between the 
two expansions in the power-law case may be the following. It is known that the Born approximation in quantum mechanics (analogous to our perturbative method with the Green's function) is good for high multipoles (angular momenta) $\ell$ of the expansion in spherical harmonics. In our approach, the parameter $\beta$ plays the role of the multipole index $\ell$ for the energy levels of the hydrogen atom, as can be seen on comparing the frequency (63) with the expression given in Ref. 23] (see also Ref. 8]). The approximation with the Green's function is therefore expected to yield more significant corrections for large values of $|\beta|$, which coincide with the regime of fast-roll. Indeed, the leading order becomes more and more accurate for increasing $|\beta|$. Since the interesting regime for inflation involves small values of $\beta \sim-2$, it is instead the adiabatic approximation which seems better since the horizon flow functions evolve slowly and/or the states are quasi-classical [1]]. This may be the reason whereby the adiabatic approximation works better in the next-to-leading order. However, in more general cases, either or both methods may contribute significant corrections. In this respect, let us remark that the adiabatic expansion can be straightforwardly applied only for a linear turning point 42], whereas the perturbative Green's function method is not so restricted.

Having assessed the accuracy of the method, it is now natural to use it in order to improve current estimates for inflationary models which are not exactly solvable. We are in fact analyzing the slow roll approximation with our method and will report about it in a future publication [33].

\section{Acknowledgments}

We would like to thank Salman Habib, Katrin Heitmann, Jerome Martin for discussions and comments on the manuscript.
[1] http://wmap.gsfc.nasa.gov

[2] http://www.sdss.org

[3] http://www.esa.int/science/planck

[4] A.D. Linde, Particle physics and inflationary cosmology (Harwood, Chur, Switzerland, 1990). A.R. Liddle and D.H. Lyth, Cosmological inflation and large-scale structure (Cambridge University Press, Cambridge, England, 2000).

[5] D. H. Lyth and E. D. Stewart, Phys. Lett. B 274, 168 (1992).

[6] E.D. Stewart and D.H. Lyth, Phys. Lett. B 302, 171 (1993).

[7] J.E. Lidsey, A.R. Liddle, E.W. Kolb, E.J. Copeland, T. Barreiro and M. Abney, Rev. Mod. Phys. 69, 373 (1997).

[8] J. Martin and D.J. Schwarz, Phys. Rev. D 67, 083512 (2003).

[9] S. Habib, K. Heitmann, G. Jungman and C. MolinaParís, Phys. Rev. Lett. 89, 281301 (2002).

[10] V.F. Mukhanov, Sov. Phys. JETP Lett. 41, 493 (1985); Sov. Phys. JETP Lett. 67, 1297 (1988).

[11] V.F. Mukhanov, H.A. Feldman and R.H. Brandenberger, Phys. Rep. 215, 203 (1992).

[12] L. Wang, V.F. Mukhanov and P.J. Steinhardt, Phys. Lett. B 414, 18 (1997).

[13] E.D. Stewart and J.O. Gong, Phys. Lett. B 510, 1 (2001).

[14] N.C. Tsamis and R.P. Woodard, Class. Q. Grav. 20, 5205 (2003).

[15] F. Finelli, G. Marozzi, G. P. Vacca, and G. Venturi, Phys. Rev. D 69, 123508 (2004).

[16] S. Habib, A. Heinen, K. Heitmann, G. Jungman and C. Molina-París, Phys. Rev. D 70, 083507 (2004).

[17] F.W.J. Olver, Asymptotics and special functions (AKP Classics, Wellesley, MA 1997).

[18] L.P. Grishchuk, Sov. Phys. JETP Lett. 40, 409 (1974).

[19] A. A. Starobinsky, JETP Lett. 30, 682 (1979).

[20] D.J. Schwarz, C.A. Terrero-Escalante and A.A. Garcìa, Phys. Lett. B 517, 243 (2001).
[21] M. Luzzi, Laurea thesis (unpublised, 2003).

[22] N.D. Birrel and P.C.W. Davies, Quantum Fields in Curved Space (Cambridge University Press, Cambridge, England, 1982).

[23] R.E. Langer, Phys. Rev. 51, 669 (1937).

[24] R.E. Langer, Bull. Am. Math. Soc. 40, 545 (1934).

[25] P. Hunt and S. Sarkar, Phys. Rev. D 70, 103518 (2004).

[26] C.M. Bender and S.A. Orszag, Advanced mathematical methods for scientists and engineers (McGraw-Hill Book Company, 1978).

[27] L.I. Schiff, Quantum Mechanics (McGraw-Hill Book Company, 1952).

[28] M. Abramowitz and I. Stegun, Handbook of mathematical functions with formulas, graphs, and mathematical table (Dover Publishing, 1965).

[29] R.E. Langer, Trans. Am. Math. Soc. 67, 461 (1949).

[30] L.F. Abbott and M.B. Wise, Nucl. Phys. B 244, 541 (1984)

[31] J. Martin and D.J. Schwarz, Phys. Rev. D 57, 3302 (1998).

[32] D. Polarski and A.A. Starobinsky, Class. Quant. Grav. 13, 377 (1996).

[33] R. Casadio, F. Finelli, M. Luzzi, G. Venturi, in progress.

[34] Note that we define $x$ with the opposite sign with respect to that in Ref. [8]. This of course implies that our $x_{i} \rightarrow$ $+\infty$ and $x_{f} \rightarrow-\infty$, whereas $x_{i} \rightarrow-\infty$ and $x_{f} \rightarrow+\infty$ in Ref. [8].

[35] Let us briefly recall that such an expansion is called adiabatic when $\sqrt{\delta}$ is the inverse of a (typically very large) time over which the system evolves slowly, and semiclassical when $\sqrt{\delta} \sim \hbar$.

[36] In the following we shall consider the general case in Eq. (23), although one might restrict to $n=1$, since our frequencies exhibit a linear turning point.

[37] Since the initial conditions must be imposed at $x$ in deep region $\mathrm{I}$, we can consider $x_{i} \sim+\infty$.

[38] The Bessel functions are actually regular around $x=x_{*}$, therefore the following expressions are just the leading 
terms in the Taylor expansion of $J_{\nu}$ and $I_{\nu}$.

[39] Since our method has some similarity with that of Ref. [16] (see also Ref. [17]), it is worth pointing out that the first order corrections shown here differ from those of Ref. [16] (at least) in that they contain contributions from both Regions I and II.

[40] The case $\beta=-2$ is singular $\left(\epsilon_{1}=0\right.$ and the expression of the scalar power spectrum blows up) and should be considered separately. However, there are no scalar per- turbations in De Sitter space-time to first order.

[41] Cosmological perturbations are amplified by inflation evolving from vacuum to highly squeezed states, which resemble classical states in the amplitude of fluctuations 32].

[42] The same limitation seems to affect the method used in Ref. [16]. 